\title{
Stressful experiences impact on pain, fatigue and mental health in people with endometriosis: a questionnaire study during the COVID-19 pandemic
}

\author{
Lysia Demetriou $^{1}$, Christian Becker ${ }^{1}$, Beatriz Martínez-Burgo ${ }^{1}$, Adriana Invitti ${ }^{2}$, Marina \\ Kvaskoff $^{3}$, Razneen Shah ${ }^{1}$, Emma Evans ${ }^{4}$, Claire Lunde ${ }^{1}$, Emma Cox ${ }^{5}$, Kurtis Garbutt ${ }^{1}$, \\ Krina Zondervan ${ }^{1}$, Elaine Fox ${ }^{1}$, and Katy Vincent ${ }^{1}$ \\ ${ }^{1}$ University of Oxford \\ ${ }^{2}$ Universidade Federal de Sao Paulo Escola Paulista de Medicina \\ ${ }^{3}$ Universite Paris-Saclay \\ ${ }^{4}$ Oxford University Hospitals NHS Foundation Trust \\ ${ }^{5}$ Endometriosis UK
}

June 29, 2021

\begin{abstract}
Objective To explore the impact of the COVID-19 pandemic on pain and fatigue symptoms and their interactions with the impact on mental health in people with endometriosis. Design Global cross-sectional survey. Setting Online survey. Sample A total of 4717 adults with a surgical or radiological diagnosis of endometriosis. Methods An online global study collected data in 5 languages between 11th May to 8th June 2020. The survey included questions on current-status and changes of endometriosis-symptoms, mental health, demographics, and the impact of the COVID-19 pandemic on the respondents' lives. Main Outcome Measures Self-reported changes to endometriosis-associated symptoms (pelvic pain, tiredness/fatigue, bleeding) and to mental health during the early stages of the COVID-19 pandemic. Results Respondents reported a marked worsening of their endometriosis symptoms (endo-associated pain $(39.3 \%$; 95\% CI [37.7, 40.5]), tiredness/fatigue (49.9\%; $95 \%$ CI [48.4, $51.2])$ and bleeding $(39.6 \% ; 95 \%$ CI $[38.2,41]))$ and mental health $(38.6 \% ; 95 \%$ CI [37.2, 39.9]). Those with a pre-existing mental health diagnosis $(38.8 \%)$ were more negatively impacted. The worsening of pain and TF were significantly correlated with worsening of mental health $(\mathrm{p}<0.001)$ and these relationships were found to be weakly mediated by pain catastrophising scores (pain: effect size: $0.071, \mathrm{LLCI}=0.060, \mathrm{ULCI}=0.082$, TF: effect size: $0.050, \mathrm{LLCI}=0.040$, ULCI $=0.060$ ). Conclusions This study demonstrates that stressful experiences impact the physical and mental health of people with endometriosis. The findings highlight the need to consider psychological approaches in the holistic management of people with endometriosis.
\end{abstract}

\section{Hosted file}

Manuscript.docx available at https://authorea.com/users/381250/articles/528384-stressfulexperiences-impact-on-pain-fatigue-and-mental-health-in-people-with-endometriosis-aquestionnaire-study-during-the-covid-19-pandemic 
Table 1. Summary of altered medications, altered planned treatments, mental health diagnosis and Covid-19 impact, presented as frequencies and percentages (\%).

\begin{tabular}{|c|c|c|}
\hline & Frequency & Percent (\%) \\
\hline Altered medication and planned treatments & 3663 & 78.4 \\
\hline Altered medication treatment & 1476 & 31.3 \\
\hline Altered planned treatment & 3151 & 66.8 \\
\hline Mental Health Diagnosis & 1831 & 38.8 \\
\hline \multicolumn{3}{|l|}{ COVID-19 Impact } \\
\hline Had symptoms of COVID-19 & 753 & 16 \\
\hline Had a positive test for COVID-19 & 79 & 1.7 \\
\hline Been admitted to hospital because of COVID-19 & 18 & 0.4 \\
\hline Has anyone you live with had symptoms of COVID-19 & 591 & 12.6 \\
\hline Has anyone you live with had a positive test for COVID-19 & 67 & 1.4 \\
\hline Has someone close to you (family or friend) died because of COVID-19 & 381 & 8.1 \\
\hline Are you considered "vulnerable"/at high risk from COVID-19 & 990 & 21.1 \\
\hline Live with someone considered "vulnerable"/at high risk from COVID-19 & 1162 & 24.7 \\
\hline Worry that endometriosis makes you more vulnerable to COVID-19 & 2534 & 53.9 \\
\hline \multicolumn{3}{|l|}{ COVID-19 pandemic impact on major life changes } \\
\hline No, nothing more than for most people & 2397 & 50.8 \\
\hline I have lost my job & 352 & 7.5 \\
\hline I have had to work much longer hours & 662 & 14 \\
\hline I have had a significant decrease in my earnings & 824 & 17.5 \\
\hline I can't run my business & 239 & 5.1 \\
\hline I have had to move out of my home & 132 & 2.8 \\
\hline My relationship with my partner has fallen apart & 241 & 5.1 \\
\hline I have had to postpone/cancel my wedding & 115 & 2.4 \\
\hline \multicolumn{3}{|l|}{ Most important/bothersome before the pandemic } \\
\hline Pelvic pain & 2602 & 55.3 \\
\hline Heavy and/or irregular bleeding & 395 & 8.4 \\
\hline Fatigue & 293 & 6.2 \\
\hline Bowel problems & 403 & 8.6 \\
\hline Urinary/bladder problems & 109 & 2.3 \\
\hline Pain during or after sex & 263 & 5.6 \\
\hline Infertility/difficulty getting pregnant & 410 & 8.7 \\
\hline
\end{tabular}


Change in mental health
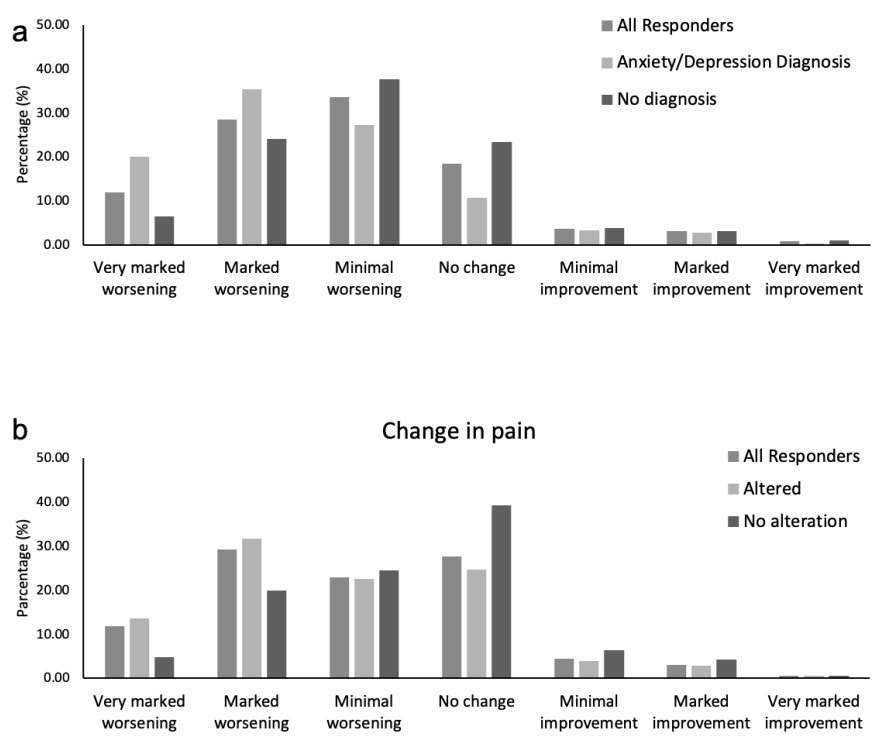

C

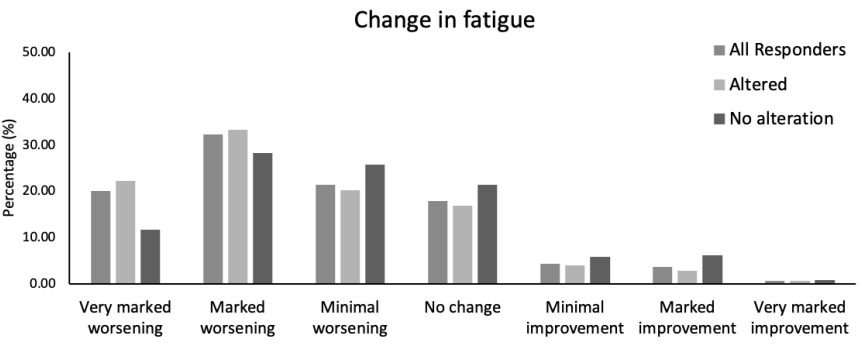


a
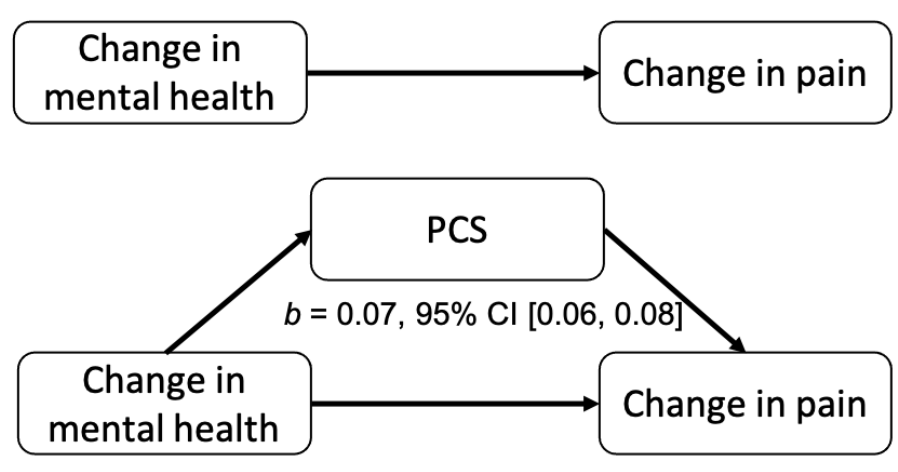

b

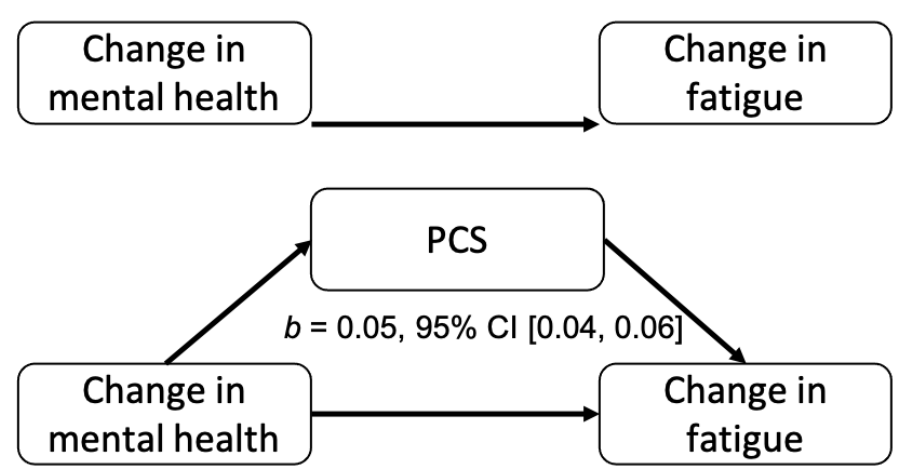

